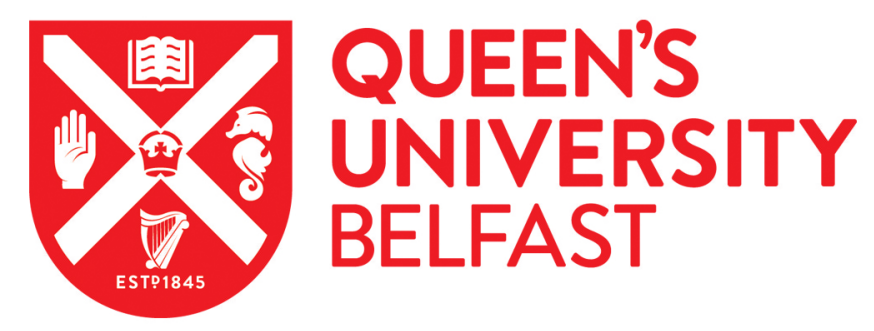

\title{
Consumers and health providers working in partnership for the promotion of person-centred health services: a co-produced qualitative evidence synthesis
}

Merner, B., Hill, S., Colombo, C., Gaulden, C., Graham-Wisener, L., Lowe, D., Walsh, L., Biggar, S., Bourke, N., Chmielewski, R., Gill, M., Martin, F., Martinek, N., McKinlay, L., Menzies, D., Mussared, A., Refahi, N., Smith, L., Sonawane, R., \& Wardrope, C. (2019). Consumers and health providers working in partnership for the promotion of person-centred health services: a co-produced qualitative evidence synthesis. Cochrane database of systematic reviews (Online). https://doi.org/10.1002/14651858.CD013274

Published in:

Cochrane database of systematic reviews (Online)

Document Version:

Publisher's PDF, also known as Version of record

Queen's University Belfast - Research Portal:

Link to publication record in Queen's University Belfast Research Portal

\section{Publisher rights}

Copyright ( 2019 The Cochrane Collaboration. Published by John Wiley \& Sons, Ltd.This work is made available online in accordance with the publisher's policies. Please refer to any applicable terms of use of the publisher.

\footnotetext{
General rights

Copyright for the publications made accessible via the Queen's University Belfast Research Portal is retained by the author(s) and / or other copyright owners and it is a condition of accessing these publications that users recognise and abide by the legal requirements associated with these rights.
}

\section{Take down policy}

The Research Portal is Queen's institutional repository that provides access to Queen's research output. Every effort has been made to ensure that content in the Research Portal does not infringe any person's rights, or applicable UK laws. If you discover content in the Research Portal that you believe breaches copyright or violates any law, please contact openaccess@qub.ac.uk. 


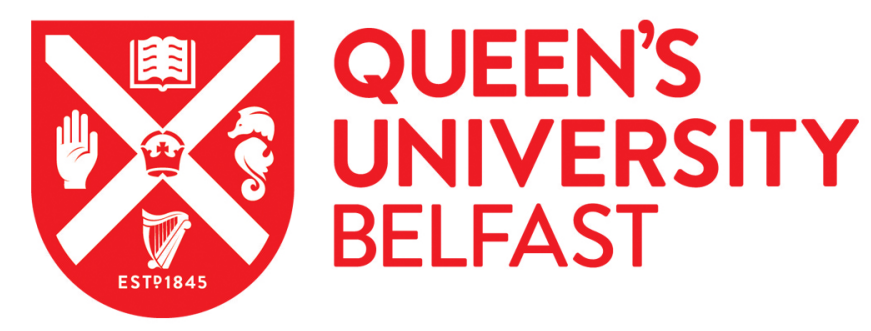

\section{Consumers and health providers working in partnership for the promotion of person-centred health services: a co-produced qualitative evidence synthesis}

Merner, B., Hill, S., Colombo, C., Gaulden, C., Graham-Wisener, L., Lowe, D., ... Wardrope, C. (2019).

Consumers and health providers working in partnership for the promotion of person-centred health services: a co-produced qualitative evidence synthesis. Cochrane database of systematic reviews (Online).

https://doi.org/10.1002/14651858.CD013274

\section{Published in:}

Cochrane database of systematic reviews (Online)

\section{Document Version:}

Publisher's PDF, also known as Version of record

Queen's University Belfast - Research Portal:

Link to publication record in Queen's University Belfast Research Portal

\section{General rights}

Copyright for the publications made accessible via the Queen's University Belfast Research Portal is retained by the author(s) and / or other copyright owners and it is a condition of accessing these publications that users recognise and abide by the legal requirements associated with these rights.

Take down policy

The Research Portal is Queen's institutional repository that provides access to Queen's research output. Every effort has been made to ensure that content in the Research Portal does not infringe any person's rights, or applicable UK laws. If you discover content in the

Research Portal that you believe breaches copyright or violates any law, please contact openaccess@qub.ac.uk. 


\section{Cochrane}

Cochrane Database of Systematic Reviews

\section{Consumers and health providers working in partnership for the promotion of person-centred health services: a co- produced qualitative evidence synthesis (Protocol)}

Merner B, Hill S, Colombo C, Xafis V, Gaulden CM, Graham-Wisener L, Lowe D, Walsh L, Biggar S, Bourke N, Chmielewski R, Gill M, Martin F, Martinek N, McKinlay L, Menzies D, Mussared A, Refahi N, Smith L, Sonawane R, Wardrope C

Merner B, Hill S, Colombo C, Xafis V, Gaulden CM, Graham-Wisener L, Lowe D, Walsh L, Biggar S, Bourke N, Chmielewski R, Gill M, Martin F, Martinek N, McKinlay L, Menzies D, Mussared A, Refahi N, Smith L, Sonawane R, Wardrope C.

Consumers and health providers working in partnership for the promotion of person-centred health services: a co-produced qualitative evidence synthesis.

Cochrane Database of Systematic Reviews 2019, Issue 2. Art. No.: CD013274.

DOI: 10.1002/14651858.CD013274.

www.cochranelibrary.com 
TABLE OF CONTENTS

HEADER . . . . . . . . . . . . . . . . . . . . . . . . . . . . . . . . . . . . . . . 1

ABSTRACT . . . . . . . . . . . . . . . . . . . . . . . . . . . . . . . . . . . . . . 1

BACKGROUND . . . . . . . . . . . . . . . . . . . . . . . . . . . . . . . . . . . . . . . . . .

OBJECTIVES . . . . . . . . . . . . . . . . . . . . . . . . . . . . . . . . . . . . . . . . . . . . . 44

METHODS . . . . . . . . . . . . . . . . . . . . . . . . . . . . . . . . . . . . . . 5

Figure 1. . . . . . . . . . . . . . . . . . . . . . . . . . . . . . . . . . . . . . 8

ACKNOWLEDGEMENTS . . . . . . . . . . . . . . . . . . . . . . . . . . . . . . . . . . . . . . . . .

REFERENCES . . . . . . . . . . . . . . . . . . . . . . . . . . . . . . . . . . . . . . . $\quad 10$

APPENDICES . . . . . . . . . . . . . . . . . . . . . . . . . . . . . . . . . . . . . . . . . . . . $\quad$.

CONTRIBUTIONS OF AUTHORS . . . . . . . . . . . . . . . . . . . . . . . . . . . . . . . . . . . . . . . . . .

DECLARATIONS OF INTEREST . . . . . . . . . . . . . . . . . . . . . . . . . . . . . . . . . . . 14

SOURCES OF SUPPORT . . . . . . . . . . . . . . . . . . . . . . . . . . . . . . . . . . . . . . . . . . .

NOTES . . . . . . . . . . . . . . . . . . . . . . . . . . . . . . . . . . . . . . . . 15

Consumers and health providers working in partnership for the promotion of person-centred health services: a co-produced qualitative

evidence synthesis (Protocol)

Copyright (c) 2019 The Cochrane Collaboration. Published by John Wiley \& Sons, Ltd. 


\title{
[Qualitative Protocol] \\ Consumers and health providers working in partnership for the promotion of person-centred health services: a co- produced qualitative evidence synthesis
}

\author{
Bronwen Merner $^{1}$, Sophie Hill ${ }^{1}$, Cinzia Colombo ${ }^{2}$, Vicki Xafis ${ }^{3,4}$, Carolyn M Gaulden ${ }^{5}$, Lisa Graham-Wisener ${ }^{6}$, Dianne Lowe ${ }^{1}$, \\ Louisa Walsh $^{1}$, Susan Biggar ${ }^{7,8}$, Noni Bourke ${ }^{9}$, Renee Chmielewski ${ }^{10}$, Marie Gill ${ }^{11}$, Fiona Martin ${ }^{12}$, Nathalie Martinek ${ }^{13}$, Louise \\ McKinlay $^{14}$, David Menzies ${ }^{15}$, Anne Mussared ${ }^{16}$, Nora Refahi ${ }^{17}$, Lorraine Smith ${ }^{18}$, Roshni Sonawane ${ }^{19}$, Cheryl Wardrope ${ }^{20}$ \\ ${ }^{1}$ Centre for Health Communication and Participation, School of Psychology and Public Health, La Trobe University, Bundoora, Aus- \\ tralia. ${ }^{2}$ Laboratory for medical research and consumer involvement, Department of Public Health, Istituto di Ricerche Farmacologiche \\ Mario Negri IRCCS, Milano, Italy. ${ }^{3}$ Centre for Biomedical Ethics, National University of Singapore, Singapore, Singapore. ${ }^{4}$ The \\ Sydney Children's Hospitals Network, Westmead, Australia. ${ }^{5}$ Detroit Wayne County Authority Health Residency Program, Michigan \\ State University, Providence Hospital, Southfield, Michigan, USA. ${ }^{6}$ School of Psychology, Queen's University Belfast, Belfast, UK. \\ ${ }^{7}$ Australian Health Practitioner Regulation Agency (AHPRA), Melbourne, Australia. ${ }^{8}$ Consumer Representative, Melbourne, Australia. \\ ${ }^{9}$ Organisational Support \& Development, Bass Coast Health, Wonthaggi, Australia. ${ }^{10}$ Planning and Patient Experience, The Royal \\ Victorian Eye and Ear Hospital, East Melbourne, Australia. ${ }^{11}$ Gill and Wilcox Consultancy, Melbourne, Australia. ${ }^{12}$ CatholicCare, East \\ Melbourne, Australia. ${ }^{13}$ Safe Space Health, Melbourne, Australia. ${ }^{14}$ Consumers as Partners, Safer Care Victoria, Melbourne, Australia. \\ ${ }^{15}$ Chronic Disease Programs, South Eastern Melbourne Primary Health Network, Heatherton, Australia. ${ }^{16}$ Consumer Representative, \\ North Belair, Australia. ${ }^{17}$ Carer Representative, Melbourne, Australia. ${ }^{18}$ School of Pharmacy, Faculty of Medicine and Health, Univer- \\ sity of Sydney, Camperdown, Australia. ${ }^{19}$ Rockingham General Hospital and Wexford Specialist Clinics, Murdoch, Australia. ${ }^{20}$ Patient \\ Safety and Quality Service, Children's Health Queensland Hospital and Health Service, South Brisbane, Australia
}

Contact address: Bronwen Merner, Centre for Health Communication and Participation, School of Psychology and Public Health, La Trobe University, Bundoora, Victoria, Australia. b.merner@latrobe.edu.au.

Editorial group: Cochrane Consumers and Communication Group.

Publication status and date: New, published in Issue 2, 2019.

Citation: Merner B, Hill S, Colombo C, Xafis V, Gaulden CM, Graham-Wisener L, Lowe D, Walsh L, Biggar S, Bourke N, Chmielewski R, Gill M, Martin F, Martinek N, McKinlay L, Menzies D, Mussared A, Refahi N, Smith L, Sonawane R, Wardrope C. Consumers and health providers working in partnership for the promotion of person-centred health services: a co-produced qualitative evidence synthesis. Cochrane Database of Systematic Reviews 2019, Issue 2. Art. No.: CD013274. DOI: 10.1002/14651858.CD013274.

Copyright (C) 2019 The Cochrane Collaboration. Published by John Wiley \& Sons, Ltd.

\section{A B S T R A C T}

This is a protocol for a Cochrane Review (Qualitative). The objectives are as follows:

Aims

- To synthesise the views and experiences of consumers and health providers of working in partnership to promote person-centred health services.

- To identify best practice principles for working in partnership at the health service level by understanding consumers' and health providers' views and experiences.

Objectives

Consumers and health providers working in partnership for the promotion of person-centred health services: a co-produced qualitative evidence synthesis (Protocol)

Copyright $₫ 2019$ The Cochrane Collaboration. Published by John Wiley \& Sons, Ltd. 
- To investigate the barriers and facilitators to working in partnership and how these may promote or impede person-centred planning, delivery or evaluation of health services. For example, if consumers are given sufficient training to help them work in partnership on a hospital quality and safety committee, they may be better able to contribute the consumer perspective to quality and safety decisions.

- To understand consumers' and health providers' views and experiences of working in partnership.

- To analyse the ways consumers and providers work in partnership (e.g. committee formats, experience-based co-design formats).

\section{B A C K G R O U N D}

\section{Introduction}

This qualitative evidence synthesis (a summary and synthesis of qualitative research, or QES) will explore consumers and health providers working in partnership for the promotion of personcentred care at the health service level.

There are many models of working in partnership and so we define our key terms first to aid understanding.

The partnerships relevant to this synthesis are complex interventions that could operate at national, state, regional or health service level.

'Working in partnership' refers to formal group formats (such as committees, councils, boards or steering groups) where the purpose is to develop strategies for planning, delivering or evaluating person-centred care at a health service level. This review will only be concerned with working in partnership where at least one consumer and health provider meet jointly and regularly to make decisions relevant to promoting person-centred care in one or more health service(s). The decisions may affect a specific part of a health service (e.g. redesigning a mental health unit), the whole health service (e.g. decision making on a hospital quality and safety committee), or a policy or programme of care affecting multiple health services (e.g. co-design of a region's maternal and child health services policy).

'Consumer' refers to a person who participates in formal partnerships in an advisory or representative capacity. This could include people who are described as consumer representatives, consumer consultants, consumers with acute or chronic conditions, carers, family members, patient representatives or members of the community or consumer organisations.

'Health provider' refers to a person who has a health policy, management, administrative, or clinical role and who participates in formal partnerships in an advisory or representative capacity. A health provider does not include a person whose primary role is a health researcher.

In particular, this QES will analyse the findings of qualitative research to explore the barriers and facilitators to working in partnership, consumers' and health providers' views of working in partnership, and the different ways that consumers and health providers partner to make decisions to promote person-centred care in health services. This QES is linked to a Cochrane effectiveness Review (a summary and synthesis of trials) titled Consumers and health providers working in partnership as an intervention for the promotion of person-centred health services (Lowe (in press)). The effectiveness review will examine the effects of interventions to promote working in partnership, which have been assessed in trials. This QES and the effectiveness review will be conducted concurrently and the implications of the findings of each will be integrated and discussed in each respectively.

\section{Person-centred care and working in partnership}

The essence of person-centred care is a healthcare system that is designed around the consumer with respect for a consumer's preferences, values and/or needs (IAPO 2007). The global move towards person-centred care in health services is a major, and relatively recent, phenomenon. Historically, medical dominance, in which health practitioners made decisions on behalf of, and without necessarily involving, their patients dominated the planning and delivery of health care (Bleakley 2014; Coulter 1999; Richards 2013). There is no agreed definition of person-centred care (ACSQHC 2011; Collins 2014; Harding 2015; IAPO 2007). The Institute for Patient- and Family-Centered Care defines person-centred care as "planning, delivery, and evaluation of health care that is grounded in mutually beneficial partnerships among healthcare providers, patients, and families" (IPFCC 2012). This definition, like many others, emphasises that partnerships with consumers underpin person-centred care. There is a similar lack of consensus regarding the principles that comprise person-centred care. The most wellknown are perhaps those defined by Picker 2018. These principles are:

- respect for consumers' values, preferences and needs;

- integration and co-ordination of care;

- information, communication and education;

- physical comfort;

- emotional support;

- involvement of family and friends;

- transition and continuity of care; and 


\section{- access to care.}

Interventions to promote person-centred care have traditionally been targeted at the clinical consultation level. The clinical consultation level refers to interactions between individual health practitioners and individual patients to plan and manage their own health care. Whilst interventions at the clinical consultation level are vital for promoting person-centred care, they are not sufficient to ensure that health care is person-centred. For example, a key principle of person-centred care, according to Picker 2018, is "transition and continuity of care". Ensuring patients receive integrated and co-ordinated care may be difficult to achieve for individual clinicians, within clinical consultations. Rather, it requires service design initiatives (involving health service managers, quality coordinators and consumer representatives) to develop systems to support individual clinicians in their practice. Other definitions and principles of person-centred care similarly reflect that personcentred care is facilitated both within and beyond the clinical consultation (see for example, IPFCC 2012; WHO 2018).

Partnering with consumers across all levels of a health service is a key facilitator in the delivery of person-centred care. A recent report from the Australian Commission on Safety and Quality in Health Care identified partnering with consumers as one of seven attributes of high performing person-centred healthcare organisations (ACSQHC 2018). Similarly, a qualitative study by Luxford 2011, which explored the facilitators and barriers to person-centred care in USA healthcare institutions, found a key facilitator was the involvement of consumers throughout a health service. These findings built on an earlier report exploring how to achieve more rapid and widespread implementation of personcentred care (Shaller 2007). Shaller 2007 found a key facilitator was the involvement of consumers at multiple levels of the organisation (including as full members of committees in the organisation). This QES will focus on partnering with consumers at the levels of health service governance and service design to improve person-centred care.

\section{The co-production of a Cochrane QES on person-centred care}

The protocol for this QES has been co-produced by researchers working with a group of Australian stakeholders in health (herein called the Stakeholder Panel) using new Cochrane co-production methods (Pollock 2015; Pollock 2017). The Stakeholder Panel consists of 18 people who have a wide range of expertise: six are consumer representatives with experience in service improvement, research or policy; six are health professionals with expertise in clinical governance; and six are involved in researching, developing or implementing quality and safety policy and standards. Panel members originally responded to an invitation to co-produce a Cochrane Review on interventions to promote person-centred care. This topic was chosen as one of the top five priority top- ics for Cochrane Consumers and Communication in its prioritysetting project (Synnot 2018). The Stakeholder Panel met twice to determine the nature, aim and scope of the review.

The Panel originally discussed whether to update an existing Cochrane Review on person-centred care, titled Interventions for providers to promote a patient-centred approach in clinical consultations (Dwamena 2012). In the Dwamena review, person-centred care (then referred to as patient-centred care) was operationalised into two core features: "1. healthcare providers share control of consultations, decisions about interventions or the management of the health problems with patients, and/or 2. healthcare providers focus on the patient as a person, rather than solely on the disease, in consultations" (Dwamena 2012). Panel members said the Dwamena effectiveness review had been used by services in the Australian health sector to inform developments in person-centred care but that person-centred care had evolved considerably since the Dwamena criteria were developed. In particular, interventions that promote person-centred care today were considered much broader in scope than those captured under the Dwamena criteria. The Panel felt there was a need for the terminology and definitions to be updated. They also indicated that different types of data to inform policy and practice, particularly qualitative research, should be considered by the review. Specifically, Panel members felt a QES was required that would examine the phenomenon of health service level interventions involving 'working in partnership' between providers and consumers, rather than focusing on the role of providers in achieving person-centred care (as examined in Dwamena 2012).

The Stakeholder Panel then voted on the type of review they would like to be taken forward (a QES, or an effectiveness review). Of the 15 Panel members who returned votes (3 did not vote), 13 preferred a QES as the best way to explore the questions raised during the Panel discussions. The researchers then scoped the options for a QES which focused on the contemporary concerns associated with the interventions for partnering between health providers and consumers.

At the next meeting, the Panel discussed the types of interventions that working in partnership may include. These included (but were not limited to) consumers as health service board members or lay governors, consumers on facility planning or infrastructure committees, consumers on safety and quality committees, patient councils and consumers involved in experience-based co-design committees. Health providers involved in partnership working may include policymakers, managers, administrators, quality managers and clinicians associated with single or multiple health services. In this context, questions raised by Panel members included: What are the barriers and facilitators to the involvement of consumers in partnership activities? What are the similarities and differences between consumers' and health providers' perceptions and experiences of person-centred care? How is an environment created which facilitates the delivery of person-centred care? Although the QES was originally planned as a stand alone review, 
Cochrane guidance only permitted the publication of QESs that were directly linked to an existing or concurrent effectiveness review (Harris 2018). Thus, it was decided an effectiveness review entitled Consumers and health providers working in partnership as an intervention for the promotion of person-centred health services (Lowe (in press)), would be undertaken in parallel with this QES. The findings of Lowe (in press) may provide information on the effects of partnership interventions in terms of health service, provider participant or consumer participant outcomes. The findings of this QES will link with the findings of the effectiveness review by providing contextual information about how or why different partnership interventions work, and may help to explain why (or not) partnering is effective for specific outcomes. The QES will also highlight issues of commonality and difference between the perceptions of health providers and consumers about person-centred care that may also be relevant to interpreting the results of the effectiveness review as well as to the implementation of personcentred approaches.

\section{Description of the phenomenon of interest}

Our Cochrane Review will explore consumers' and health providers' experiences of working in partnership to promote person-centred care. We will use the IPFCC 2012 definition to define person-centred care: "planning, delivery, and evaluation of health care that is grounded in mutually beneficial partnerships among healthcare providers, patients, and families".

The partnerships relevant to this synthesis are complex interventions that involve consumers and health providers working together in formal group formats (such as committees, councils, boards or steering groups) to make decisions relevant to personcentred care in single or multiple health services. The purpose of working in partnership is associated with the planning, delivery or evaluation of any aspect of a health service. This could include operational improvements as well as strategic planning. For example, working in partnership for the planning of person-centred health services could include consumer representation on governance committees, community advisory committees and patient councils. Working in partnership for person-centred delivery of health services could include experience-based co-design of services and co-production of health information. And finally, working in partnership for the evaluation of health services could include consumer representation on quality and safety committees and involvement in patient experience or feedback committees. Decisions taken while working in partnership may impact on the whole of the organisation (e.g. policies for service delivery) or have more distal impacts on the care provided to individual patients (e.g. health information provided for patients with asthma).

Firstly, the QES will investigate the barriers and facilitators to working in partnership and how these may promote or impede person-centred planning, delivery and evaluation of health services. Barriers and facilitators of working in partnership from the con- sumer perspective may revolve around roles, selection and skills, support and training (Edgman-Levitan 2013; HCCA 2017). Barriers and facilitators from a provider perspective may include executive support, time, and role clarity (Edgman-Levitan 2013; McCoy 2012; Mockford 2012). Barriers to both working together include attitudes of respect and awareness of what each party may bring and how to harness differing abilities or skills (Edgman-Levitan 2013; McCoy 2012; Mockford 2012; Nathan 2011; WHO 2010).

Secondly, the QES will explore consumers' and health providers' views and experiences of partnership. This is necessary because health providers and consumers may have different perceptions of person-centred care (Dwamena 2012; Gillespie 2004; Kreindler 2013). Further, working in partnership is a phenomenon that should be examined in different social, cultural and health system contexts because beliefs, social norms, regulations as well as the social determinants of health, can impact on whether, and how, partnering occurs ( Edgman-Levitan 2013; Mockford 2012; McCoy 2012).

Thirdly, the QES will explore the range of ways that consumers partner with health providers at the health service level when working towards the delivery of person-centred care. For example, Taylor 2015 conducted semi-structured interviews with hospital managers, expert country informants (people who had countrywide expertise in understanding the background and current policy themes in person-centred care), patient organisations and a consumer representative from hospitals across Europe. The findings showed person-centred care was a relatively new concept in some European countries and, as such, seeking patient feedback was a key approach to person-centred care at the health service level. Other European countries, in which person-centred care was more established and developed used approaches that incorporated the patient's voice as an integral part of management decisions (Taylor 2015).

\section{Why it is important to conduct this review}

This review will contribute to building Cochrane's evidence base for and understanding of the phenomenon of promoting personcentred care.

This is a burgeoning area in policy, and health services are seeking guidance informed by evidence, as the input from the Stakeholder Panel demonstrated.

\section{O B JE C T I VES}

\section{Aims}


- To synthesise the views and experiences of consumers and health providers of working in partnership to promote personcentred health services.

- To identify best practice principles for working in partnership at the health service level by understanding consumers' and health providers' views and experiences.

\section{Objectives}

- To investigate the barriers and facilitators to working in partnership and how these may promote or impede personcentred planning, delivery or evaluation of health services. For example, if consumers are given sufficient training to help them work in partnership on a hospital quality and safety committee, they may be better able to contribute the consumer perspective to quality and safety decisions.

- To understand consumers' and health providers' views and experiences of working in partnership.

- To analyse the ways consumers and providers work in partnership (e.g. committee formats, experience-based co-design formats).

\section{METHODS}

\section{Criteria for considering studies for this review}

\section{Types of phenomena of interest}

We will include qualitative studies of the phenomenon of working in partnership for the promotion of person-centred health services. Person-centred care is: "planning, delivery, and evaluation of health care that is grounded in mutually beneficial partnerships among healthcare providers, patients, and families" (IPFCC 2012).

Working in partnership is defined as "healthcare organisations, healthcare providers and policy makers actively working with people who use the healthcare system to ensure that health information and services meet people's needs" (ACSQHC 2018).

For the purposes of this review, working in partnership will be operationalised as consumers and health providers meeting jointly and regularly in formal group formats to share decision making for the purpose of promoting person-centred care in one or more health service(s).

To be eligible for inclusion, studies must include the following features.

- Consumers and health providers are engaged in formal group formats, such as committees, councils, boards or steering groups.
- The group involves at least one consumer and at least one health provider.

- The group meets jointly, more than once via face-to-face or electronic modes.

- The group may be ongoing or time-limited (for example, groups formed for a specific project).

- The group shares responsibility for decision making for planning and/or delivery and/or evaluation of person-centred health services.

We will exclude studies which involve partnering with consumers for decision making about an individual's care or treatment. We will exclude these because they focus on individual consultations, rather than health services. We will also exclude studies concerned with partnering with consumers for health services research (planning, undertaking or disseminating research), including a health service's management of research (research funding panels, setting research priorities, research ethics and governance). We will exclude these studies because they focus on research in health services rather than health service planning, delivery and evaluation. We will include studies which involve partnering with consumers for health provider education, but only when these partnerships include formal group formats (e.g. a training committee).

\section{Types of studies}

This review will include primary, empirical qualitative studies that include a description of the sampling strategy, data collection procedures and the type of data analysis undertaken (Hannes 2011). We will also include the qualitative component of mixed methods studies. We will exclude opinion pieces, vignettes and editorials. We will search the included, excluded and ongoing studies in the linked effectiveness review by Lowe (in press) for eligible qualitative trial sibling studies.

\section{Types of settings}

The setting for outcomes of the partnering approaches will be health services. For the purposes of this review, health services are defined as healthcare organisations that provide direct care to patients in primary settings (e.g. community health centre, general practitioner practice), secondary settings (e.g. specialist outpatient clinics), or tertiary settings (e.g. hospitals). We will include person-centred care relevant to home and residential services only when they focus primarily on providing health or nursing care (e.g. home-based nursing services, nursing homes, residential rehabilitation services or hospices). Studies from rural and urban settings will be eligible for inclusion, as will those from high-income, middle-income, and low-income countries.

\section{Types of participants}


'Consumer' refers to a person who participates in formal partnerships in an advisory or representative capacity. This could include people who are described as consumer representatives, consumer consultants, consumers with acute or chronic conditions, carers, family members, patient representatives or members of the community or consumer organisations.

'Health provider' refers to a person who has a health policy, management, administrative, or clinical role and who participates in formal partnerships in an advisory or representative capacity. A health provider does not include a person whose primary role is a health researcher.

We will exclude health provider participants who take on the role of consumers, as their primary role is to represent providers rather than consumers.

We will separately analyse data from consumer and health provider participants, when possible. We will include the findings of studies when the views of consumers or health providers are combined, as long as the findings address the objectives of this review. Where consumer or provider data are combined with other types of participants (e.g. community development officers), we will include these studies. We will not include the views of other participants, such as stakeholders external to the partnership activity.

\section{Search methods for identification of studies}

\section{Electronic searches}

Our scoping searches of this topic show a proliferation of studies about partnering with consumers published after 2000. The concept of person-centred care, particularly in terms of the health service level, has also developed greatly since 2000. As the aim of the synthesis is to build knowledge and conceptual depth, rather than to provide an exhaustive synthesis that includes every identifiable study, we will be searching the databases below from 2000 onwards.

- MEDLINE (OvidSP) (2000 to present).

- Embase (OvidSP) (2000 to present).

- PsycINFO (OvidSP) (2000 to present).

- CINAHL (EBSCO) (2000 to present).

We present the strategy for MEDLINE (OvidSP) in Appendix 1. This search has been developed by the Information Specialist for Cochrane Consumers and Communication and is informed by searching guidelines from the Cochrane Qualitative and Implementation Methods Group (Harris 2018).

We will tailor the strategy to other databases and report them in the review.

There will be no geographic restrictions. Due to the challenges associated with translating qualitative research articles, and the resources required for translation, we will select only English-language articles.

\section{Searching other databases}

We will search relevant grey literature sources, such as ProQuest Theses and Dissertations. Using key words, we will also search the websites of key national and international organisations involved in promoting person-centred care, including The King's Fund, The Health Foundation, National Institute for Health and Care Excellence, Planetree, Picker Institute Europe, Institute for Health Improvement, World Health Organization, Australian Commission on Safety and Quality in Health Care, and the Agency for Healthcare Research and Quality.

We will contact experts in the field and authors of sampled studies for advice as to other relevant studies. We will also search reference lists of sampled studies and relevant systematic reviews.

\section{Data collection and analysis}

\section{Selection of studies}

\section{Data extraction and management}

Two review authors will independently screen all titles and abstracts identified from searches to determine which meet the inclusion criteria. We will retrieve in full text any papers identified as potentially relevant by at least one review author. Two review authors will independently screen full text articles for inclusion or exclusion, with discrepancies resolved by discussion and by consulting a third review author, if necessary, to reach consensus. We will list all potentially relevant papers excluded from the review at this stage as excluded studies, with reasons provided in the 'Characteristics of excluded studies' table. We will also collate and report details of duplicate publications, so that each study (rather than each report) is the unit of interest in the review. We will report the screening and selection process in an adapted PRISMA flow chart (Liberati 2009).

If we identify more than 30 to 40 eligible studies, we will use a purposive sampling approach to select the most information-rich studies for analysis (Patton 2002). Purposive sampling of 30 to 40 included studies has been used successfully in a previous Cochrane QES Review (Ames 2017). Maximum variation sampling will be the type of purposive sampling used in this review. Maximum variation sampling involves selecting a wide range of studies to get variation on particular dimensions of the phenomenon (Patton 2002). The particular dimensions of interest in answering the review question will be the richness of the data, geographical location, setting (e.g. hospital or community clinics), participants (including underserved or vulnerable consumer groups, and a range of health providers), and type of partnering (e.g. consumer-majority committees versus consumer-minority committees). Two review authors will independently select the studies to be included in the sample. Their selections will be compared and any discrepancies 
will be resolved through discussion until consensus is reached, or through consultation with a third review author, when necessary. All papers that meet the inclusion criteria, but are not sampled, will be listed in a separate table. The table will include the reason each paper was not sampled.

One review author will extract data independently from the sampled studies. A second review author will check the data extraction. Any discrepancies will be resolved by discussion until consensus is reached, or through consultation with a third review author, where necessary. We will develop and pilot a data extraction form adapted from Cochrane Consumers and Communication's Data Extraction Template (available at: cccrg.cochrane.org/author-resources) and the NICE 2012 Examples of Evidence Tables for Qualitative Studies. We will extract the following data: details of the study (methodology, data collection and analysis methods, setting, geographic location), details of the participants (number and description of participants, methods of recruitment, sampling and inclusion criteria), and details of the study findings. In each sampled study, we will consider text describing the results or findings of the study 'results data' for the purposes of the synthesis (Thomas 2015). All extracted data will be checked for accuracy against the data extraction sheets by a second review author working independently. Data will be entered into Review Manager 5 (Review Manager 2014), (the software used for preparing and maintaining Cochrane Reviews) by one review author and checked for accuracy by a second review author.

\section{Appraisal of study quality}

We will appraise the quality of each included study using the Critical Appraisal Skills Programme (CASP) tool (CASP 2018). We chose the CASP because it provides for the assessment of the following domains of methodological strengths and limitations: clear aims and research question, congruence between the aims and the research design, rigour of sampling and data collection to address the question, and appropriate application of the method (Noyes 2018). One of the review authors with qualitative methods experience will apply the CASP to each sampled study. A second review author, also with qualitative methods experience, will check each appraisal for discrepancies. Any disagreements will be resolved through discussion or consultation with a third review author. We will not exclude any studies on the basis of quality because this may result in the loss of valuable insights (Hannes 2011). However, the appraisal will form part of the review authors' judgements when conducting the GRADE-CERQual assessment (Lewin 2018; described below), which determines how much confidence we have in each finding of the synthesis.

\section{Data synthesis}

We will use thematic synthesis as the method for synthesising the findings of the primary qualitative studies (Thomas 2015). All results data from the sampled studies will be entered verbatim into NVivo by one review author, with a second review author checking the data entry (NVivo 2014). Analysis of the data will occur in four stages: 1 . undertake initial coding, 2. develop descriptive themes, 3. draft analytical themes, and 4. finalise analytical themes. These stages are shown in Figure 1. 
Figure I. Stages of data synthesis

\section{Stage 1: Undertake initial coding}

- At least two review authors independently code data from sampled studies in units of meaning (initial codes)

- At least two review authors compare codes and resolve discrepancies (with involvement of third review author when needed)

- At least two review authors compare and contrast data with the same initial code to check if additional codes are needed

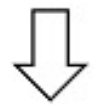

\section{Stage 2: Develop descriptive themes}

- At least two review authors will group similar initial codes under 'descriptive codes'

- At least two review authors will group similar descriptive codes under 'descriptive themes'

- One review author will draft a summary of qualitative findings table for each descriptive theme, and a second review author will check and comment on the draft

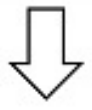

\section{Stage 3: Draft analytical themes}

- 3 review authors will independently infer how the descriptive themes relate to the review questions

- All review authors will meet to discuss inferences and emerging abstract/theoretical themes, as well as disconfirming cases

- Draft analytical themes applied to review questions and amended as required

- Repeat this stage until analytical themes include all descriptive themes and address specific review questions

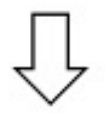

\section{Stage 4: Finalise analytical themes}

- Finalise analytical themes through consensus between review authors and stakeholder panel 
Stage 1 Undertake initial coding: during this stage, at least two review authors will independently code the data in units of meaning. The codes will be compared and any discrepancies will be resolved through discussion until consensus is reached, or through consultation with a third review author, when necessary. Data with the same initial code will be compared and contrasted to check whether interpretation is consistent across the existing code or whether additional codes are needed (Thomas 2015).

Stage 2 Develop descriptive themes: at least two review authors will categorisesimilar initial codes under a new 'descriptive' code that captures the meaning of the group. We will then organise these codes under 'descriptive themes'. Once we have developed the descriptive themes, one of the review authors will draft a 'Summary of Qualitative Findings' table for each descriptive theme listing the constituent descriptive codes and the studies comprising each desciptive code. Another review author will examine the draft and provide comments until agreement on a final draft is agreed ( Thomas 2015).

Stage 3 Drafting analytical themes: in this stage, we will apply the descriptive themes explicitly to the review questions. At least three review authors will independently infer how the descriptive themes of the synthesis address the questions explicitly asked in the review (Thomas 2015). These questions are as follows.

- What are the barriers and facilitators to working in partnership and how do these promote or impede person-centred planning, delivery or evaluation of health services?

- How do consumers and health providers view and experience working in partnership?

- What are the ways that consumers and health providers work in partnership?

Review authors (including authors who have not been involved previously in the analysis) will then meet to discuss these individual inferences, leading to the emergence of more abstract, or theoretical themes. During the discussion, review authors will be encouraged to actively search for deviant or disconfirming cases, for example, specific subgroups for whom there are unique barriers or facilitators (Booth 2013). The review authors will then reexamine the review questions in light of these themes and changes will be made as needed. This final stage of the process will be repeated until the theoretical themes describe all of the descriptive themes, as well as address the specific review questions (Thomas 2015).

Stage 4 Finalise analytical themes: in this stage, we will present the draft analytical themes to the Panel for their input. The themes will be finalised through a process of consensus between the review authors and the Stakeholder Panel.

\section{Assessment of confidence in the review findings}

We will use the GRADE-CERQual approach to assess confidence in the review findings (Lewin 2018). GRADE-CERQual assesses the extent to which the findings of the review are representative of the phenomenon being explored (Lewin 2018). The assessment is based on four components: the methodological limitations of the studies contributing to the finding; the relevance to the review question of the studies contributing to the review finding; the adequacy of the data supporting the review finding; and the coherence of the review finding (Lewin 2015). After making judgements about these four components, we will give each finding an overall rating of confidence as high, moderate, low or very low. We will apply the GRADE-CERQual assessment to each review finding through discussion by at least two review authors working collaboratively. We will present a 'CERQual evidence profile' for each finding. This will include the assessment for each CERQual component, the overall CERQual assessment, and the studies contributing to each finding. The 'Summary of Qualitative Findings' tables will also include the overall confidence assessment for each finding, and the justification of the confidence assessment.

\section{Using the synthesised qualitative findings to supplement the Cochrane effectiveness Review}

We will use a sequential approach to integrate the findings of our QES with the findings of the concurrent Lowe (in press) effectiveness review (Harden 2018). The findings of the QES will help to identify contextual factors relevant to the effectiveness of interventions for working in partnership to promote person-centred care. The QES may also assist in identifying outcomes relevant to partnerships and to delivery of person-centred care that are important to both providers and consumers. We will finalise the aims and method of integration of the findings after the thematic synthesis is completed. One option is to explore whether the findings from this QES (e.g. contextual information about how or why different partnership interventions work) can be linked to the effects of interventions on outcomes assessed in the accompanying Cochrane effectiveness Review (e.g. helping to explain why (or not) partnering is effective for specific outcomes) via a logic model. If we choose a logic model as the integration method, at least two review authors will develop it. We will then present the draft to the Stakeholder Panel for their views on applicability and transferability and we will integrate their feedback.

\section{Author reflexivity}

As an author team, we will reflect on our individual backgrounds and values with a view to understanding how these may affect our collection and analysis of the data. We will share the results of the synthesis and the draft of the logic model with the Stakeholder 
Panel as a way of potentially identifying any assumptions that may be influencing the synthesis.

\section{Relevance to consumers}

We have outlined how the co-design of this protocol for a QES has proceeded and involved the Stakeholder Panel. Members of the Panel will be directly involved in the production of the review through participation in the screening and data extraction processes. We will discuss the findings from the included studies at a face-to-face meeting with the Stakeholder Panel in order to develop the final themes for the synthesis of findings. We will also ask the Panel members for their input on the potential implications of the results for their own fields of practice. All members of the Panel have contributed suggested organisations for the dissemination stage to ensure the protocol and review will reach consumer, health provider, research and policy networks.

With this QES, we aim to inform consumers, clinicians and relevant personnel in health services on the implementation of partnering including the assistance, training and support which consumers or staff may require.

\section{ACKNOWLEDGEMENTS}

The authors gratefully acknowledge the input and guidance of the Stakeholder Advisory Panel formed for this review. The Australian-based Stakeholder Advisory Panel consists of consumers, healthcare professionals and health policy makers. The members of the Panel are: Leslie Arnott (Consumer Representative, Victoria), Susan Biggar (Consumer Representative, Victoria), Noni
Bourke (Executive Director Quality and Risk, Bass Coast Health, Victoria), Paul Bryden (Consumer Representative, Queensland), Renee Chmielewski (Manager, Planning and Patient Experience, The Royal Victorian Eye and Ear Hospital, Victoria), Leia Earnshaw (Consumer Representative, Australian Capital Territory), Marie Gill (Gill and Wilcox consultancy, Victoria), Fiona Martin (Clinical Practice Lead/Health Psychologist, CatholicCare, Melbourne-Gippsland), Nathalie Martinek (Safe Space Health, Wellbeing Consultant for Health Professionals, Victoria), Louise McKinlay (Director, Consumers as Partners, Safer Care Victoria), David Menzies (Manager, Chronic Disease Programs, South Eastern Melbourne Primary Health Network, Victoria), Nancy Messino (Clinical Trials Quality Officer, Victorian Comprehensive Cancer Centre, Victoria), Anne Mussared (Consumer Representative, South Australia), Naomi Poole (Program Manager, Partnering with Consumers, Australian Commission on Safety and Quality in Health Care), Nora Refahi (Consumer Representative, Victoria), Lorraine Smith (Professor, School of Pharmacy, Faculty of Medicine and Health, University of Sydney, NSW), Roshni Sonawane (Consultant Paediatrician, Rockingham General Hospital and Wexford Specialist clinics, Murdoch, Western Australia) and Cheryl Wardrope (Consumer Engagement Officer, Patient Safety and Quality Service, Children's Health Queensland Hospital and Health Service, South Brisbane, Queensland).

The author team acknowledge the guidance from Professor Jane Noyes on QES methods.

The author team would also like to thank Rebecca Ryan, contact editor from Cochrane Consumers and Communication, for her guidance in developing this protocol.

\section{R E F E R E N C E S}

\section{Additional references}

\section{ACSQHC 2011}

Australian Commission on Safety and Quality in Health Care (ACSQHC). Patient-centred care: improving quality and safety through partnerships with patients and consumers. www.safetyandquality.gov.au/wp-content/ uploads/2012/03/PCC_Paper_August.pdf. Sydney: ACSQHC, 2011.

\section{ACSQHC 2018}

Australian Commission on Safety and Quality in Health Care (ACSQHC). Review of key attributes of highperforming person-centred healthcare organisations. www.safetyandquality.gov.au/wp-content/uploads/2018/ 08/FINAL-REPORT-Attributes-of-person-centredhealthcare-organisations-2018.pdf. Sydney, 2018.

Ames 2017

Ames HM, Glenton C, Lewin S. Parents' and informal caregivers' views and experiences of communication about routine childhood vaccination: a synthesis of qualitative evidence. Cochrane Database of Systematic Reviews 2017, Issue 2. DOI: 10.1002/14651858.CD011787.pub2

Booth 2013

Booth A, Carroll A, Ilott C, Low LL, Cooper K. Desperately seeking dissonance: Identifying the disconfirming case in qualitative evidence synthesis. Qualitative Health Research 2013;1:126-41.

\section{CASP 2018}

Critical Appraisal Skills Programme (CASP). Making sense of evidence: 10 questions to help you make sense of qualitative research. casp-uk.net/wp-content/uploads/2018/ 03/CASP-Qualitative-Checklist-2018_fillable_form.pdf. Oxford (UK): Public Health Resource Unit, 2018.

\section{Collins 2014}

Collins A, The Health Foundation. Measuring what really matters: towards a coherent measurement system to support 
person-centred care. www.health.org.uk/sites/default/files/ MeasuringWhatReallyMatters.pdf 2014.

\section{Dwamena 2012}

Dwamena F, Holmes-Rovner M, Gaulden CM, Jorgenson

S, Sadigh G, Sikorskii A, et al. Interventions for providers to promote a patient-centred approach in clinical consultations. Cochrane Database of Systematic Reviews 2012, Issue 12. DOI: 10.1002/14651858.CD003267.pub2

Edgman-Levitan 2013

Edgman-Levitan S, Brady C, Howitt P, Patient and Family Engagement Working Group. Partnering with Patients, Families, and Communities for Health: A Global Imperative. www.imperial.ac.uk/media/imperialcollege/institute-of-global-health-innovation/public/ 27425_WISH_Patient-Engagement_web.pdf 2013.

\section{Gillespie 2004}

Gillespie R, Florin D, Gillam S. How is patient-centred care understood by the clinical, managerial and lay stakeholders responsible for promoting this agenda?. Health Expectations 2004;7(2):142-8.

\section{Hannes 2011}

Hannes K. Chapter 4: Critical appraisal. In: Noyes J, Booth A, Hannes K, Harden A, Harris J, Lewin S, et al. editor(s). Supplemental Handbook Guidance for Inclusion of Qualitative Research in Cochrane Systematic Reviews of Interventions. Version 1 (updated August 2011). London (UK): Cochrane Collaboration Qualitative Methods Group, 2011.

\section{Harden 2018}

Harden A, Thomas J, Cargo M, Harris J, Pantoja T, Flemming K, et al. Cochrane Qualitative and Implementation Methods Group guidance series - paper 5: methods for integrating qualitative and implementation evidence within intervention effectiveness reviews. Journal of Clinical Epidemiology 2018;97:70-8. DOI: 10.1016/ j.jclinepi.2017.11.029

\section{Harding 2015}

Harding E, Wait S, Scrutton J, The Health Policy Partnership. The state of play in person-centred care: a pragmatic review of how person-centred care is defined, applied and measured. www.healthpolicypartnership.com/ wp-content/uploads/State-of-play-in-person-centred-carefull-report-Dec-11-2015.pdf 2015.

\section{Harris 2018}

Harris JL, Booth A, Cargo M, Hannes K, Harden A, Flemming K, et al. Cochrane Qualitative and Implementation Methods Group guidance series-paper 2: methods for question formulation, searching, and protocol development for qualitative evidence synthesis. Journal of Clinical Epidemiology 2018;97:39-48. DOI: 10.1016/ j.jclinepi.2017.10.023

\section{HCCA 2017}

Health Care Consumers Association. Of course it's better if we're there: consumer involvement in health infrastructure in the ACT, 2009-2016. www.hcca.org.au/wp-content/ uploads/2018/04/Consumer-involvement-in-health-
infrastructure-projects-in-the-ACT-2009-2016-FINALDECEMBER-2016.compressed.pdf 2017.

\section{IAPO 2007}

International Alliance of Patients' Organizations (IAPO). What is patient-centred healthcare? A review of definitions and principles. iapo.org.uk/sites/default/files/files/ IAPO $\% 20$ Patient-Centred $\% 20$ Healthcare $\% 20$ Review $\% 20$ 2nd\%20edition.pdf (accessed 8 October 2018).

\section{IPFCC 2012}

Institute for Patient- and Family-Centered Care. What is PFCC?. www.ipfcc.org/about/pfcc.html (accessed prior to 25 November 2018). [http://www.ipfcc.org/about/ pfcc.html]

\section{Kreindler 2013}

Kreindler S. The politics of patient-centred care. Health Expectations 2013;18(5):1139-50.

\section{Lewin 2015}

Lewin S, Glenton C, Munthe-Kaas H, Carlsen B, Colvin $\mathrm{CJ}$, Gülmezoglu $\mathrm{M}$, et al. Using qualitative evidence in decision making for health and social interventions: An approach to assess confidence in findings from qualitative evidence syntheses (GRADE-CERQual). PLoS Medicine 2015;12(10):e1001895.

\section{Lewin 2018}

Lewin S, Booth A, Glenton C, Munthe-Kaas H, Rashidian A, Wainwright M, et al. Applying GRADE-CERQual to qualitative evidence synthesis findings: introduction to the series. Implementation Science 2018;13(Suppl 1):2.

Liberati 2009

Liberati A, Altman DG, Tetzlaff J, Mulrow C, Gøtzsche PC, Ioannidis JP, et al. The PRISMA statement for reporting systematic reviews and meta-analyses of studies that evaluate health care interventions: explanation and elaboration. PLoS Medicine 2009;6:e1000100.

Lowe (in press) Lowe D, Hill S, Merner B, Walsh L, Graham-Wisener L. Consumers and health providers working in partnership as an intervention for the promotion of person-centred health services. Cochrane Database of Systematic Reviews (in press).

\section{Luxford 2011}

Luxford K, Safran DG, Delbanco T. Promoting patientcentered care: A qualitative study of facilitators and barriers in healthcare organizations with a reputation for improving the patient experience. International Journal for Quality in Health Care 2011;23(5):510-5. DOI: 10.1093/intqhal mzr024

\section{McCoy 2012}

McCoy DC, Hall JA, Ridge M. A systematic review of the literature for evidence on health facility committees in lowand middle-income countries. Health Policy and Planning 2012;27(6):449-66.

\section{Mockford 2012}

Mockford C, Staniszewska S, Griffiths F, Herron-Marx S. The impact of patient and public involvement on UK NHS 
health care: a systematic review. International Journal for Quality in Health Care 2012;24(1):28-38.

\section{Nathan 2011}

Nathan S, Johnston L, Braithwaite J. The role of community representatives on health service committees: Staff expectations vs. reality. Health Expectations 2011;14 (3):272-84.

\section{NICE 2012}

National Institute for Health Care Excellence (NICE). Methods for the development of NICE public health guidance (3rd edition, 2012). www.nice.org.uk/process/ pmg4/chapter/introduction (accessed 8 October 2018).

\section{Noyes 2018}

Noyes J, Booth A, Flemming K, Garside R, Harden A, Lewin S, et al. Cochrane Qualitative and Implementation Methods Group guidance series-paper 3: Methods for assessing methodological limitations, data extraction and synthesis, and confidence in synthesized qualitative findings. Journal of Clinical Epidemiology 2018;97:49-58. DOI: 10.1016/j.jclinepi.2017.06.020

NVivo 2014 [Computer program]

QSR International Pty Ltd. NVivo qualitative data analysis. Version 10. Melbourne: QSR International Pty Ltd, 2012.

Patton 2002

Patton MQ. Qualitative Research and Evaluation Methods. 3rd Edition. Thousand Oaks (CA): Sage Publications, 2002.

Picker 2018

Picker. Picker principles of person-centered care. www.picker.org/about-us/picker-principles-of-personcentred-care/ (accessed 8 October 2018).

\section{Pollock 2015}

Pollock A, Campbell P, Baer G, Choo Pl, Morris J, Forster A. User involvement in a Cochrane systematic review: using structured methods to enhance the clinical relevance, usefulness and usability of a systematic review update. Systematic Reviews 2015;4:55. DOI: 10.1186/ s13643-015-0023-5

Pollock 2017

Pollock A, Morley R, Watts C. Involving people: A learning resource for systematic review authors. training.cochrane.org/involving-people. London: Cochrane Training, (accessed 8 October 2018).
Review Manager 2014 [Computer program]

Nordic Cochrane Centre, The Cochrane Collaboration. Review Manager 5 (RevMan 5). Version 5.3. Copenhagen: Nordic Cochrane Centre, The Cochrane Collaboration, 2014 .

Ryan 2016

Ryan R, Horey D, Oliver S, McKenzie J, Prictor M, Santesso $\mathrm{N}$, et al. Cochrane Consumers and Communication Group standard protocol text and additional guidance for review authors (2016). cccrg.cochrane.org/author-resources (accessed 8 October 2018).

Shaller 2007

Shaller D. Patient-centered care: what does

it take?. www.commonwealthfund.org/

sites/default/files/documents/me-

dia_files_publications_fund_report_2007_oct_patient_centered_care_what_does_it_take_(accessed 8 October 2018).

\section{Synnot 2018}

Synnot A, Bragge P, Lowe D, Nunn JS, O'Sullivan M, Horvat $\mathrm{L}$, et al. Research priorities in health communication and participation: international survey of consumers and others. BMJ Open 2018;8(5):e019481.

\section{Taylor 2015}

Taylor A, Groene O. European hospital managers' perceptions of patient-centred care: A qualitative study on implementation and context. Journal of Health Organization and Management 2015;29(6):711-28. DOI: 10.1108/ JHOM-11-2013-0261

\section{Thomas 2015}

Thomas J, Harden A. Methods for the thematic synthesis of qualitative research in systematic reviews. $B M C$ Medical Research Methodology 2008;8:45. DOI: 10.1186/ 1471-2288-8-45

\section{WHO 2010}

World Health Organization (WHO). Peoplecentred care in low- and middle-income countries. www.personcenteredmedicine.org/doc/genevathree/ geneva2011i.pdf (accessed 8 October 2018).

\section{WHO 2018}

World Health Organization (WHO). Health systems service delivery. www.who.int/healthsystems/topics/delivery/en/. Geneva: WHO, (accessed prior to 25 November 2018).

* Indicates the major publication for the study 


\section{A P P E N D I C E S}

\section{Appendix I. MEDLINE search strategy}

1. exp Community Participation/

2. Stakeholder Participation/

3. Decision Making/

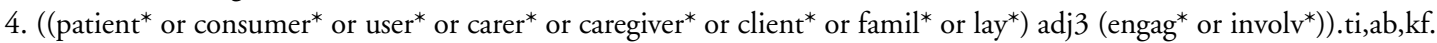

5. or/1-4

6. "Health Priorities"/

7. exp Patient Care Team/

8. exp Ambulatory Care Facilities/

9. *"Mental Health Services"/

10. *"Community Health Services"/

11. * "Health Services Administration"/

12. "Quality Improvement"/

13. *"Hospitals, Public"/

14. "Quality of Health Care"/

15. "Delivery of Health Care"/

16. * Delivery of Health Care, Integrated"/

17. or/6-16

18. "Community-Institutional Relations"/

19. * "Advisory Committees"/og

20. (partner* or participat* or consult* or decision* or deliberation* or co\#design* or involv* or contribut* or role* or empower* or engag* or collab* or advoca* or organi\#ation* or respons*).ti,ab,kf.

21. (experience based adj2 design).ti,ab,kf.

22. or/ $18-20$

23. interview:.ti,ab.

24. interview/

25. experience*.tw.

26. qualitative.ti,ab.

27. or/23-26

28. 5 and 17 and 22

29. 27 and 28

30. limit 29 to $\mathrm{yr}=$ "2000 -Current"

\section{CONTRIBUTIONSOFAUTHORS}

BM - conceptual development of the protocol, drafting of the protocol, organising and participating in stakeholder advisory panel meetings

$\mathrm{SH}$ - conceptual development of the protocol, chairing of stakeholder advisory panel meetings, writing and providing feedback on drafts

CC - conceptual development of the protocol, participating in stakeholder advisory panel meetings, providing feedback on drafts

VX - conceptual development of the protocol, participating in stakeholder advisory panel meetings, providing feedback on drafts

CG - conceptual development of the protocol, providing feedback on drafts

LGW - conceptual development of the protocol, providing feedback on drafts

$\mathrm{DL}$ - conceptual development of the protocol, providing feedback on drafts

LW - development of data extraction template, providing feedback on drafts

Consumers and health providers working in partnership for the promotion of person-centred health services: a co-produced qualitative 
SB - stakeholder panel member, providing feedback on drafts

$\mathrm{NB}$ - stakeholder panel member, providing feedback on drafts

$\mathrm{RC}$ - stakeholder panel member, providing feedback on drafts

MG - stakeholder panel member, providing feedback on drafts

NM - stakeholder panel member, providing feedback on drafts

FM - stakeholder panel member, providing feedback on drafts

LM - stakeholder panel member, providing feedback on drafts

DM - stakeholder panel member, providing feedback on drafts

AM - stakeholder panel member, providing feedback on drafts

NR - stakeholder panel member, providing feedback on drafts

LS - stakeholder panel member, providing feedback on drafts

RS - stakeholder panel member, providing feedback on drafts

$\mathrm{CW}$ - stakeholder panel member, providing feedback on drafts

\section{DECLARATIONSOF INTEREST}

BM - received funding from La Trobe University to lead the review; Managing Editor of the Cochrane Consumers and Communication Group. BM was not involved in the editorial process for this review

SH - Co-ordinating Editor of the Cochrane Consumers and Communication Group. SH was not involved in the editorial process for this review

CC - none known

VX - none known

CG - none known

LGW - none known

DL - Technical editor of the Cochrane Consumers and Communication Group. DL was not involved in the editorial process for this review

LW - none known

$\mathrm{SB}$ - none known

NB - none known

$\mathrm{RC}$ - none known

MG - none known

FM - none known

NM - none known

LM - Safer Care Victoria (my employer) oversees the contract and funds provided to the Centre for Health Communication amd Participation at La Trobe University which supports this Cochrane Review. The contract itself is administered by my department in Safer Care Victoria. This has no bearing in my view on this work.

DM - none known

AM - none known

Consumers and health providers working in partnership for the promotion of person-centred health services: a co-produced qualitative 
NR - none known

LS - none known

RS - none known

$\mathrm{CW}$ - none known

\section{SOURCES OF SUPPORT}

\section{Internal sources}

- La Trobe University, Australia.

$\mathrm{BM}$ receives funding from La Trobe University to undertake this review

\section{External sources}

- No sources of support supplied

\section{NOTES}

This protocol includes standard text and guidance provided by Cochrane Consumers and Communication (Ryan 2016). 